\title{
Acceptability of oral
} iodinated contrast media: a head-to-head comparison of four media

\section{A POLLENTINE, MB BChir, FRCR, E NGAN-SOO, MB BS and P MCCOUBRIE, MB BS, FRCR}

Department of Radiology, Southmead Hospital, Bristol, UK

Address correspondence to: Dr Adrian Pollentine

E-mail: adrianpollentine@doctors.org.uk

Objective: To assess the palatability of iodinated oral contrast media commonly used in abdominopelvic CT and CT colonography (CTC).

Methods: 80 volunteers assessed the palatability of a 20-ml sample of a standard $30 \mathrm{mg} \mathrm{ml}^{-1}$ dilution of Omnipaque (iohexol; GE Healthcare, Cork, Ireland), Telebrix ${ }^{\circledast}$ (meglumine ioxithalamate; Guerbet, Aulnay-sous-Bois, France), Gastromiro $^{\circledast}$ (iopamidol; Bracco, High Wycombe, UK) and Gastrografin (sodium diatrizoate and meglumine diatrizoate; Bayer, Newbury, UK) in a computer-generated random order.

Results: Gastrografin is rated significantly less palatable than the remaining media $(p<0.005)$. Omnipaque and Telebrix are significantly more palatable than Gastromiro.
No difference existed between Omnipaque and Telebrix. $39 \%$ of participants would refuse to consume the quantities of Gastrografin required for a CTC examination compared with Telebrix (7\%) and Omnipaque (9\%) $(p<0.05)$.

Conclusion: Omnipaque and Telebrix are significantly more palatable than both Gastromiro and Gastrografin, with participants more willing to ingest them in larger quantities as well as being less expensive.

Advances in knowledge: Omnipaque and Telebrix are significantly more palatable iodinated oral contrast media than both Gastromiro and Gastrografin, which has potential implications in compliance with both abdominopelvic CT and CTC. 
Oral-iodinated contrast media are widely used in fluoroscopic gastrointestinal examinations, diagnostic abdominopelvic CT and, more recently, CT colonography (CTC). The traditional administration of positive oral contrast media in fluoroscopy and diagnostic CT allows characterisation of the bowel wall and differentiates it from surrounding structures, whereas in CTC oral contrast media "tag" faecal matter with high-density iodine. This allows for the confident differentiation of faecal material from mucosal pathology by virtue of its high attenuation. Thus, "faecal tagging" allows a reduction in the amount of bowel catharsis necessary for a diagnostic study. Indeed, it is quite possible to use an oral iodinated contrast medium alone with good results-so-called "laxative-free" preparation $[1,2]$.

This approach increases patient acceptability and willingness to undergo the examination $[3,4]$, as cathartic bowel preparation has been shown to be the most burdensome aspect of the investigation [5-8]. Furthermore, it is safer to use bowel preparation that minimises fluid and electrolyte shifts, particularly in those with liver, renal or cardiac disease [9].

Despite the advances and improvements in techniques that have been made to improve the patient experience for abdominal CT and CTC, patients are still required to ingest relatively large volumes of oral contrast media for bowel opacification and faecal tagging [10]. These contrast media are frequently described as noxious-tasting substances and regularly associated with nausea and vomiting [11]. In our experience, this unpleasant taste is the principal reason why patients often do not fully comply with the required bowel preparation. A simple way to potentially increase compliance is to ensure that contrast media are as palatable as possible.

The primary purpose of this study is to assess the palatability of four iodinated contrast media commonly used as enteric contrast and faecal tagging media. Three of the media examined in this study are used in routine clinical practice within our local region. The last medium, Telebrix Gastro ${ }^{\circledR}$ (Guerbet, Aulnay-sous-Bois, France), is used extensively within Europe and Canada $[12,13]$ and has recently been granted an import licence to the UK. The four media investigated were as follows: Gastrografin ${ }^{\circledR}(100 \mathrm{mg}$ sodium diatrizoate and $660 \mathrm{mg}$ meglumine diatrizoate per ml; Bayer, Newbury, UK),
Gastromiro $^{\circledR}$ (612.4 mg iopamidol per $\mathrm{ml}$; Bracco, High Wycombe, UK), Omnipaque ${ }^{\circledR} 300 \quad(647 \mathrm{mg}$ iohexol per ml; GE Healthcare, Cork, Ireland) and Telebrix Gastro (660 mg meglumine ioxithalamate per $\mathrm{ml}$ ).

\section{METHODS}

Participants were healthy volunteers aged 18 years or older, recruited from the radiology department staff at our institution. Participants were excluded if they were pregnant, had a previous history of renal disease, had a history of adverse reaction to iodine or had a concurrent ailment that would impact on their ability to assess the palatability of the contrast media (e.g. sinusitis or a cold). Participants gave oral informed consent to take part in the study and partook willingly without financial remuneration.

Ethics approval was applied for and waived by our institutional ethics advisory committee.

Participants were instructed to refrain from eating, drinking or smoking for an hour before the test and to avoid use of strong perfumes or fragrances.

Each contrast medium was anonymised and labelled A, B, C or D. The contrast media were diluted with mineral water to a standard concentration of $30 \mathrm{mg}$ iodine per $\mathrm{ml}$, which is the standard dilution used for faecal tagging in our institution [14]. 20-ml samples were prepared from these pre-mixed jugs in clear 200-ml plastic cups, ensuring an identical volume and appearance of each contrast medium. The study took place in a closed environment, with only the study co-ordinator and a maximum of two volunteers at a time. Participants were instructed not to discuss the contrast media before, during or following the study until the last results had been collected.

Each participant was asked to assess a $20-\mathrm{ml}$ sample of each diluted contrast medium in a computer-generated random order. Before tasting each contrast medium, the participant ingested a sip of mineral water, a small square of unsalted cracker followed by a further sip of water in order to cleanse and neutralise the palate fully between each medium.

After sampling each contrast medium, participants were asked to rate the palatability on both a $100-\mathrm{mm}$ visual analogue scale (VAS) and a seven-point Likert scale. The 
Table 1. Summary of the mean and standard deviations of the visual analogue scale (VAS) scores of each of the contrast media with median and interquartile ranges for the Likert responses

\begin{tabular}{|l|c|c|c|c|}
\hline Palatability scores & Omnipaque & Telebrix & Gastromiro & Gastrografin \\
\hline Mean taste VAS $(\mathrm{mm})$ & $54(20)$ & $50(22)$ & $44(25)$ & $30(23)$ \\
\hline $\begin{array}{l}\text { Median taste Likert scale } \\
\text { (interquartile range) }\end{array}$ & $4(4-5)$ & $4(3-5)$ & $3(2.5-5.0)$ & $2(1-4)$ \\
\hline
\end{tabular}

VAS ranged from "worst tasting" (0) to "best tasting" (100) at the lower and upper ends of the scale, respectively. The Likert scale ranged from "dislike very much" (1) to "like very much" (7). Participants were allowed to alter their scores at the end of the tasting, allowing them to rate all four media in context to the others. At the end of the tasting, participants were asked to rank the palatability of the media in order of preference from best tasting to worst tasting.

Finally, the participants were asked if they would be willing to drink three $200-\mathrm{ml}$ servings of each of the contrast media (yes, no or unsure) with a meal over a 48-h period as part of bowel preparation for CTC as per our institution's current bowel preparation protocol [14].

\section{STATISTICAL ANALYSIS}

For all statistical comparisons, any difference associated with a two-tailed $p$-value of $<0.05$ was considered statistically significant. Data that were not normally distributed, including the Likert scale assessment, were analysed using Wilcoxon's signed rank test. Continuous data are presented as means with standard deviations and comparison between groups performed using Student's $t$-test using a Bonferroni correction for multiple group comparison. The willingness to consume larger volumes of each medium was analysed using Fisher's exact test. Gender distribution was analysed by using the $\chi^{2}$ test.

\section{RESULTS}

80 people undertook the taste test. $56 \%$ were female and $44 \%$ were male (no statistical difference).

Significant differences were found between the palatability of the four contrast media, with Omnipaque rated the most palatable and Gastrografin rated the least. Summary of the mean and standard deviations of the VAS scores of each of the media with median and interquartile ranges for the Likert responses are shown in Table 1. Box plots showing their distribution are shown in Figure 1.

Pairwise comparisons between the four solutions are shown in Table 2. Omnipaque is rated the most palatable, but this is not significantly more so than Telebrix on either the VAS or the Likert scale. Gastrografin is rated significantly worse than all the other media $(p<0.005)$. Telebrix is rated significantly better than Gastromiro on the Likert scale but not on the VAS. Omnipaque is significantly more palatable than both Gastromiro and Gastrografin.

Participants were asked to rank the media from most to least palatable, with $43 \%$ rating Omnipaque as best tasting compared with $26 \%$ for Telebrix, $20 \%$ for Gastromiro and 11\% for Gastrografin. 68\% of people rated Gastrografin as the worst tasting medium. The most common (mode) ranking order was Omnipaque best followed by Telebrix then Gastromiro, with Gastrografin rated worst.

Participants were asked whether they would drink larger quantities of each medium as part of bowel preparation for CTC. The proportion of people answering "yes"

Figure 1. Box plots demonstrating median and interquartile ranges of the Likert scale scores for the four contrast media.

Likert scale box plot

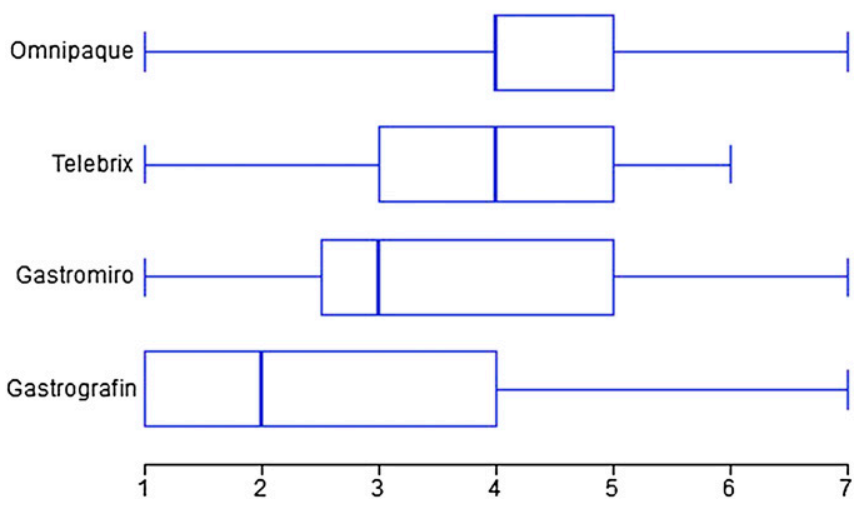


Table 2. Pairwise comparisons among the four solutions with mean, $95 \%$ confidence intervals (Cls) and the level of statistical significance

\begin{tabular}{|l|c|c|c|}
\hline Comparison & Mean & 95\% CI & $p$-value \\
\hline Gastrografin-Omnipaque & -24.2 & -28.9 to -19.5 & $<0.005$ \\
\hline Gastrografin-Telebrix & -20.0 & -25.0 to -14.9 & $<0.005$ \\
\hline Gastrografin-Gastromiro & -14.2 & -19.5 to -8.9 & $<0.005$ \\
\hline Gastromiro-Omnipaque & -10.0 & -15.0 to -5.1 & $<0.05$ \\
\hline Gastromiro-Telebrix & -5.8 & -11.0 to -0.6 & 0.12 \\
\hline Telebrix-Omnipaque & -4.3 & -8.9 to 0.4 & 0.2 \\
\hline
\end{tabular}

was $82 \%$ for Omnipaque, $80 \%$ for Telebrix, $75 \%$ for Gastromiro and 39\% for Gastrografin. Significantly fewer participants would be happy to ingest Gastrografin than to ingest the other three media $(p<0.005)$. The proportion answering "no" was 39\% for Gastrografin and $19 \%$ for Gastromiro, which is statistically higher $(p<0.005)$ than for the remaining media, with $9 \%$ and $7 \%$ unwilling to consume larger volumes of Omnipaque and Telebrix, respectively. No immediate side effects were observed.

\section{DISCUSSION}

Few studies have directly compared the palatability of multiple oral contrast media and even fewer with particular reference to those contrast media used in CTC.

We have assessed four contrast media in a head-to-head taste test, deliberately choosing those that are commonly used both for bowel opacification in abdominal $\mathrm{CT}$ and for faecal tagging in CTC. We have set out to compare in as controlled and rigorous a way as possible the palatability of these frequently prescribed media.

We feel that the taste of these media is an important factor to consider and is particularly pertinent given the wide range of clinical conditions in which these patients attend the radiology department, from the acutely unwell inpatient to the relatively asymptomatic outpatient. It seems logical that one way to potentially improve compliance and the patient experience across the board is to use a contrast medium that is as palatable as possible.

We have found a significant preference for Omnipaque and Telebrix, with Gastrografin being the least popular contrast medium. Our findings have confirmed those of previous studies comparing palatability of contrast media in the context of abdominopelvic CT. These have also demonstrated significant patient preference for Telebrix compared with Gastrografin [15-17], with no significant difference in side effects or diagnostic performance. Our study has also replicated previous findings, demonstrating a significant proportion of patients unwilling to drink Gastrografin again compared with Telebrix [17]. A study by Rollandi et al [18] demonstrated significantly greater palatability and ease of ingestion and significantly less diarrhoea and vomiting with Gastromiro than with Gastrografin. Further studies have shown patient preference for Omnipaque than for Gastrografin [19].

The contrast media we have investigated as well as differing in terms of their taste also differ in terms of their osmolality. Gastrografin and Telebrix are both hyperosmolar contrast media with concentrations of $1.94 \mathrm{osmol} \mathrm{kg}^{-1}$ and $1.89 \mathrm{osmol} \mathrm{kg}^{-1}$, respectively. To our knowledge, they are the only licensed hyperosmolar oral contrast media available. Gastromiro and Omnipaque, on the other hand, are low-osmolality media, with osmolality closer to that of plasma at $0.58 \mathrm{osmol} \mathrm{kg}^{-1}$ and $0.67 \mathrm{osmol} \mathrm{kg}^{-1}$, respectively. Hyperosmolar contrast media hold a theoretical advantage in the context of CTC as they tend to resist colonic fluid absorption, aiding stool softening, allowing the contrast medium to mix and incorporate more effectively with the retained material and increasing the homogeneity of tagging [12]. Homogeneously tagged residue aids reader confidence in differentiating mucosal pathology from the residual material. This has additional potential implications in electronic bowel cleansing and computeraided detection applications. The potential downside of this effect in terms of abdominopelvic CT is an increase in diarrhoea experienced by patients [20]. Conversely, this cathartic side effect is beneficial in the setting of CTC, reducing the volume of colonic residue and 
resulting in a "cleaner" colon. This has allowed some investigators to institute reduced-cathartic bowel preparation regimes combining satisfactory image quality and good patient tolerability $[1,12,14]$. There remains a paucity of evidence regarding the efficacy of low-osmolality contrast media in the context of CTC. The cathartic properties of hyperosmolar iodinated contrast media have also been shown to have clinical benefits in the management of adhesive small bowel obstruction, reducing the operative rate as well as the time for resolution of symptoms [21].

Cost of contrast media is an important factor to consider when assessing their use in routine abdominal CT and CTC, being an integral part of tests whose use continues to expand year on year. These costs are institution and region specific, and manufacturers are unwilling for definitive unit costs of their products to be published in this arena. On the basis of the unit cost of a 100-ml bottle of contrast medium as negotiated by our trust, Omnipaque is the cheapest followed by Telebrix then Gastrografin, with Gastromiro being the most expensive.

Thus, in our study, Omnipaque and Telebrix have been shown to have advantages in palatability and cost over the remaining two contrast media. Our participants have also demonstrated relative willingness to ingest the larger quantities of these two contrast media that would be required for CTC preparation compared with Gastromiro and Gastrografin.
Our study has several limitations. The study was designed solely to assess the palatability of contrast media and as such the diagnostic performance of the media has not been evaluated, although previous work has demonstrated no significant differences in the context of abdominal CT [15-19]. Furthermore, Telebrix has been shown to be a perfectly adequate tagging-only bowel preparation medium [2]. Further study is required to assess whether the inherent higher osmolality of Telebrix and Gastrografin confers an advantage in the context of CTC as discussed previously. Our study population comprised healthy volunteers and not a patient group. As such, results such as $39 \%$ of participants stating they would not take the required volumes of Gastrografin for CTC preparation may be higher than in symptomatic patient groups experiencing concerning symptoms requiring investigation. However, the difference between those unwilling to take Gastrografin and those unwilling to take Omnipaque/Telebrix is significant and takes on greater importance in the context of an asymptomatic screening population.

\section{CONCLUSION}

Omnipaque and Telebrix are significantly more palatable than both Gastromiro and Gastrografin, with participants more willing to ingest them in larger quantities as well as being less expensive. The inherent high osmolality of Telebrix and Gastrografin may confer an advantage over low-osmolality contrast media in the context of CTC, with further work necessary to establish this.

\section{REFERENCES}

1. Iafrate F, Hassan C, Zullo A, Stagnitti A, Ferrari R, Spagnuolo A, et al. CT colonography with reduced bowel preparation after incomplete colonoscopy in the elderly. Eur Radiol 2008;18: 1385-95. doi: 10.1007/s00330-0080892-2.

2. Liedenbaum M, Denters M, Zijta F, van Ravesteijn V, Bipat S, Vos F, et al. Reducing the oral contrast dose in CT colonography: evaluation of faecal tagging quality and patient acceptance. Clin Radiol

2011;66:30-7. doi: 10.1016/

j.crad.2010.06.016.

3. Taylor SA, Halligan S, Burling P, Bassett P, Bartram CI. Intraindividual comparison of patient acceptability of multidetector-row CT colonography and double-contrast barium enema. Clin Radiol 2005; 60:207-14. doi: 10.1016/ j.crad.2004.07.006.

4. Mahgerefteh S, Fraifeld S, Blachar A, Sosna J. CT colonography with decreased purgation: balancing preparation, performance and patient acceptance. AJR Am J Roentgenol 2009;193:1531-9. doi: 10.2214/AJR.09.2342.

5. Harewood G. A prospective, controlled asessment of factors influencing acceptance of screening colonoscopy. Am J Gastroenterol 2002;97:3186-94. doi: 10.1111/ j.1572-0241.2002.07129.x.

6. Ristvedt SL, McFarland EG, Weinstock LB, Thyssen EP. Patient 
preferences for CT colonography, conventional colonoscopy and bowel preparation. Am J Gastroenterol 2003;98:578-85.

7. Svensson MH, Svensson E, Lasson A, Hellström M. Patient acceptance of CT colonography and conventional colonoscopy: prospective comparative study in patients with or suspected of having colorectal disease. Radiology 2002;222:337-45.

8. Gluecker TM, Johnson CD, Harmsen WS, Offord KP, Harris AM, Wilson LA, et al. Colorectal cancer screening with $\mathrm{CT}$ colonography, colonoscopy and double-contrast barium enema examination: prospective assessment of patient perspectives and preferences. Radiology 2003;227:378-84.

9. Wexner SD, Beck DE, Baron TH, Fanelli RD, Hyman N, Shen B, et al. A consensus document on bowel preparation before colonoscopy: prepared by a task force from American Society of Colon and Rectal Surgeons (ASCRS), American Society for Gastrointestinal Endoscopy (ASGE), and Society of American Gastrointestinal and Endoscopic Surgeons (SAGES). Surg Endosc 2006;63:894-909.

10. Hara AK, Kuo MD, Blevins M, Chen MH, Yee J, Dachman A, et al. National CT colonography trial (ACRIN 6664): comparison of three full-laxative bowel preparations in more than 2500 average-risk patients. AJR Am J
Roentgenol 2011;196:1076-82. doi: 10.2214/AJR.10.4334.

11. Iannaccone R, Laghi A, Catalano C, Mangiapane F, Lamazza A, Schillaci A, et al. Computed tomographic colonography without cathartic preparation for the detection of colorectal polyps. Gastroenterology 2004;127:1300-11.

12. Liedenbaum $\mathrm{MH}$, de Vries $\mathrm{A}$, Gouw CI, van Rijn AF, Bipat S, Dekker E, et al. CT colonography with minimal bowel preparation: evaluation of tagging quality, patient acceptance and diagnostic accuracy in two iodine-based preparation schemes. Eur Radiol 2010;20:367-76. doi: 10.1007/ s00330-009-1570-8.

13. Canadian Association of Radiologists. CAR CT colonography standards. Ottawa, ON: CAR; 2010 [cited January 2010]. Available from: http://www.car.ca/uploads/ standards\%20guidelines/201001_ CAR_CTC_Standards_EN.pdf

14. Pollentine A, Mortimer A, McCoubrie P, Archer L. Evaluation of two minimal-prep regimes for CT colonography: optimising image quality and patient acceptability. Br J Radiol 2012;85:1085-92. doi: 10.1259/bjr/22421731.

15. Stabler A, Fink U, Siuda S, Neville $S$. Inter-individual testing of watersoluble oral contrast media in respect of diagnostic ranking, side effects and taste. [In German.] Rontgenblatter 1989;42:224-7.
16. van Waes PF, Feldberg MA, Barth P. Comparison of Telebrix Gastro and Gastrografin in abdominal computed tomography. Eur J Radiol 1989;9:179-81.

17. Bach D. Telebrix: a better tasting oral contrast agent for abdominal computed tomography. Can Assoc Radiol J 1991;42:98-101.

18. Rollandi G, Curone P, Pastorino C, Fanti B, Martinoli C, Bonati F, et al. Gastromiro vs Gastrografin vs Prontobarium-CT for computed tomography. Multicentre study. Radiol Med 1991;82:295-302.

19. Diederichs G, Franiel T, Asbach P, Romano V, Hamm B, Rogalla P. Oral administration of intravenous contrast media: a tasty alternative to conventional contrast media in computed tomography. Rofo 2007; 179:1061-7.

20. Zalis ME, Perumpillichira JJ, Magee C, Kohlberg G, Hahn PF. Tagging-based, electronically cleansed CT colonography: evaluation of patient comfort and image readability. Radiology 2006;239:149-59. doi: 10.1148/radiol.2383041308.

21. Di Saverio S, Catena F, Ansaloni L, Gavioli M, Valentino M, Pinna AD. Water-soluble contrast medium (Gastrografin) value in adhesive small intestine obstruction (ASIO): a prospective, randomized, controlled, clinical trial. World J Surg 2008;32:2293-304. doi: 10.1007/ s00268-008-9694-6. 